\title{
Specific Point-of-Care Testing of Coagulation in Patients Treated with Dabigatran
}

\author{
Florian Härtig ${ }^{1,2}$ Ingvild Birschmann ${ }^{3}$ Andreas Peter ${ }^{4,5,6}$ Matthias Ebner ${ }^{1,2,7}$ Charlotte Spencer ${ }^{1,2}$ \\ Michael Gramlich ${ }^{8}$ Hardy Richter ${ }^{1,2}$ Joachim Kuhn ${ }^{3}$ Rainer Lehmann ${ }^{4,5,6}$ Gunnar Blumenstock ${ }^{9}$ \\ Christine S. Zuern ${ }^{8,10}$ Ulf Ziemann ${ }^{1,2}$ Sven Poli ${ }^{1,2}$
}

\author{
${ }^{1}$ Department of Neurology \& Stroke, Eberhard Karls University of \\ Tübingen, Tübingen, Germany \\ 2 Hertie Institute for Clinical Brain Research, Eberhard Karls \\ University of Tübingen, Tübingen, Germany \\ 3 Institute for Laboratory and Transfusion Medicine, Heart and \\ Diabetes Center, Ruhr University, Bad Oeynhausen, Germany \\ ${ }^{4}$ German Centre for Diabetes Research (DZD), Neuherberg, \\ Germany \\ ${ }^{5}$ Institute for Diabetes Research and Metabolic Diseases, Helmholtz \\ Centre Munich, University of Tübingen, Tübingen, Germany \\ ${ }^{6}$ Institute for Clinical Chemistry and Pathobiochemistry, \\ Department for Diagnostic Laboratory Medicine, Tübingen, \\ Germany
}

Address for correspondence Sven Poli, MD, Department of Neurology \& Stroke, University Hospital Tübingen, Hoppe-Seyler-Str. 3, 72706 Tübingen, Germany (e-mail: sven.poli@uni-tuebingen.de).

${ }^{7}$ Department of Internal Medicine and Cardiology, Campus Virchow Hospital, Charité, Berlin, Germany

${ }^{8}$ Department of Cardiology, University Hospital Tübingen, Germany

${ }^{9}$ Department of Clinical Epidemiology and Applied Biometry, University of Tübingen, Tübingen, Germany

${ }^{10}$ Department of Cardiology and Cardiovascular Research Institute Basel, University Hospital Basel, University of Basel, Basel, Switzerland

Thromb Haemost 2021;121:782-791.

\begin{abstract}
Keywords

- dabigatran

- point-of-care testing

- ecarin clotting time

- thrombolysis

- anticoagulation
\end{abstract}

Background and Purpose Accurate and rapid assessment of coagulation status is necessary to guide thrombolysis or reversal of anticoagulation in stroke patients, but commercially available point-of-care (POC) assays are not suited for coagulation testing in patients treated with direct oral anticoagulants (DOACs). We aimed to evaluate the direct thrombin monitoring (DTM) test card by Helena Laboratories (Texas, United States) for anti-lla-specific POC coagulation testing, hypothesizing that its POC-ecarin clotting time (POC-ECT) accurately reflects dabigatran plasma concentrations.

Methods A prospective single-center diagnostic study (ClinicalTrials.gov-identifier: NCT02825394) was conducted enrolling patients receiving a first dose of dabigatran and patients already on dabigatran treatment. Blood samples were collected before drug intake and $0.5,1,2,8$, and 12 hours after intake. POC-ECT was performed using whole blood (WB), citrated blood (CB), and citrated plasma (CP). Dabigatran plasma concentrations were determined by mass spectrometry.

Results In total, 240 blood samples from 40 patients contained 0 to $275 \mathrm{ng} / \mathrm{mL}$ of dabigatran. POC-ECT with WB/CB/CP ranged from 20 to 186/184/316 seconds. Pearson's correlation coefficient showed a strong correlation between dabigatran concentrations and POC-ECT with WB/CB/CP $\left(R^{2}=0.78 / 0.90 / 0.92\right)$. Dabigatran concentrations $>30$ and $>50 \mathrm{ng} / \mathrm{mL}$ (thresholds for thrombolysis, surgery, and reversal therapy according to clinical guidelines) were detected by POC-ECT with WB/CB/CP (>36/35/ received

September 29, 2020 accepted after revision November 8, 2020 published online January 14, 2021
DOI https://doi.org/ $10.1055 / \mathrm{s}-0040-1721775$. ISSN 0340-6245.

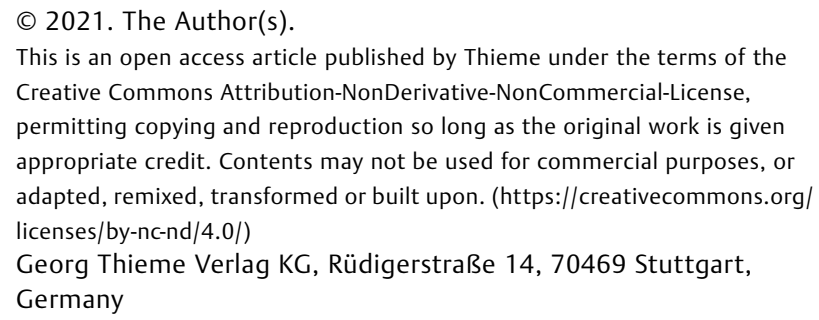


45 and $>43 / 45 / 59$ seconds) with $95 / 97 / 97$ and $96 / 98 / 97 \%$ sensitivity, and $81 / 87 / 94$ and $74 / 60 / 91 \%$ specificity.

Conclusion This first study evaluating DOAC-specific POC coagulation testing revealed an excellent correlation of POC-ECT with actual dabigatran concentrations. Detecting clinically relevant dabigatran levels with high sensitivity/specificity, the DTM assay represents a suitable diagnostic tool in acute stroke, hemorrhage, and urgent surgery.

\section{Introduction}

In patients treated with anticoagulants, the coagulation status must be assessed in the emergency situation when relevant alterations of hemostasis would represent a contraindication for intravenous thrombolysis in the case of ischemic stroke or an indication for reversal therapy in the case of major/intracranial hemorrhage or prior to urgent surgery.

For patients treated with vitamin $\mathrm{K}$ antagonists (VKAs), commercially available point-of-care (POC) testing systems that measure prothrombin time/international normalized ratio (INR) have proven their reliability in doing so significantly faster as compared with laboratory-based assays, thus shortening the interval between admission and effective therapy. ${ }^{1,2}$

In a rising number of patients treated with direct oral anticoagulants (DOACs), rapid and accurate determination of suitable coagulation parameters remains a challenge: laboratory-based DOAC-specific assays-such as anti-Xa activity for Xa inhibitors and antithrombin (Ila) activity (i.e., diluted thrombin time, ecarin clotting time [ECT], or chromogenic assays) for dabigatran-have long turnaround times and are still widely unavailable. ${ }^{3}$ Commercially available POC tests may be able to provide information about moderately elevated DOAC concentrations; their diagnostic performance at low DOAC levels, however, is limited. ${ }^{4-6}$

In this study, we evaluated the direct thrombin monitoring (DTM) test card on a Cascade Abrazo POC device (both Helena Laboratories, Beaumont, Texas, United States). Neither U.S. Food and Drug Administration approval nor the European CE mark has been obtained for the DTM test cards so far. The DTM assay measures ECT and was originally devised to monitor the anti-Ila activity of bivalirudin in patients undergoing catheter-based coronary intervention. We hypothesized that POC-ECT may be suitable to accurately determine dabigatran plasma concentrations in a blood sample. To our knowledge, this study represents the first clinical evaluation of a DOAC-specific POC assay.

\section{Methods}

Anonymized source data and study protocol will be made available to other researchers on request to the last author.

\section{Standard Protocol Approvals}

Independent review board approval was obtained prior to all study-related activity from the Ethics Committee of
Tübingen University (protocol no. 270/2015BO1). The trial was registered at Clinicaltrials.gov under the unique identifier number NCT02825394.

\section{Setting and Eligibility}

The study was single-center prospective diagnostic study with blinded outcome assessment. The study was conducted at the Department of Neurology and Department of Cardiology at Tübingen University Hospital, a tertiary care facility in Germany. We planned to include 40 patients either receiving first doses of dabigatran or being on continuous dabigatran treatment (20 in each group). Patients being treated with other anticoagulants (VKA within 14 days, other DOAC within 7 days, low-molecular-weight heparins [LMWHs] within 24 hours, or unfractionated heparin [UFH] within 12 hours prior to enrolment), spontaneously altered coagulation (INR $>1.2$, activated partial thromboplastin time $>37$ seconds), or known coagulopathy were excluded.

\section{Sample Collection and Measurements}

Venous blood samples were taken from every patient at six prespecified time points: before intake of the first or a regular dabigatran dose, 30 minutes, 1, 2, 8, and 12 hours after intake.

Whole blood (WB) was drawn directly into a noncitrated, nonheparinized syringe (Injekt, B. Braun, Melsungen, Germany) and used to conduct POC-ECT within 15 seconds of sampling; additional blood was drawn into a standard blood sampling tube for coagulation assays (S-Monovette Citrate 3.2\%, Sarstedt, Nümbrecht, Germany) to yield citrated blood (CB). POC-ECT was conducted with CB and-after centrifugation (at 2,500 g for 15 minutes)- with the supernatant citrated plasma (CP).

Chromogenic Biophen Direct Thrombin Inhibitor (BDTI, Hyphen BioMed, Neuville-sur-Oise, France) assay, calibrated to yield estimated dabigatran plasma concentrations in the standard range of 0 to $500 \mathrm{ng} / \mathrm{mL}$, was performed on a Siemens Sysmex CS5100 instrument (Siemens Healthcare Diagnostics, Eschborn, Germany).

Global coagulation tests as well as full blood count, electrolytes, inflammatory markers, protein/albumin, liver, and kidney function tests were performed at baseline.

$\mathrm{CP}$ samples from each time point were stored at $-80^{\circ} \mathrm{C}$ at our center, and later shipped to the Institute for Laboratory and Transfusion Medicine at the Heart and Diabetes Centre of the Ruhr University (Bad Oeynhausen, Germany) for ultraperformance liquid chromatography/tandem mass spectrometry (UPLC-MS/MS), which was performed in a manner 
previously described as the gold-standard method for exact determinationof dabigatran plasma concentrations. ${ }^{7}$ Shortly, mass spectrometric measurements of dabigatran were performed by monitoring the fragmentation of the single-charged molecular ion (dabigatran $+\mathrm{H}^{+}$) with a transition of $m / z 472.2$ $\rightarrow 289.2$ for quantification of the drug. The corresponding $\left[{ }^{13} \mathrm{C}_{6}\right]$-isotope $\left(\left[{ }^{13} \mathrm{C}_{6}\right.\right.$-dabigatran $+\mathrm{H}^{+}$, transition of $m / z 478.2$ $\rightarrow$ 295.2) was used as internal standard. Furthermore, a second transition of the single-charged dabigatran molecular ion $(\mathrm{m} / \mathrm{z}$ $472.2 \rightarrow 306.2$ ) and a second transition of the single-charged internal standard ion $(m / z 478.2 \rightarrow 312.2)$ were monitored for qualification to detect interferences, which may be present in the complex biological matrix and which could be interfered with measurement accuracy. In addition to UPLC-MS/MS, batch analysis of calibrated ECT was performed using the STA-ECA II assay (Diagnostica Stago, Asnieres-sur-Seine, France) on a Siemens BCS system (Siemens Healthcare Diagnostics, Eschborn, Germany).

All POC and laboratory-based tests were performed according to manufacturers' instructions by thoroughly trained investigators and technicians.

\section{Determination of POC-ECT}

For POC-ECT measurements we used DTM test cards, provided by Helena Laboratories, on a Cascade Abrazo analyzer. Underneath its transparent cover, the test card's reaction chamber contains ecarin, calcium, buffers, and (paramagnetic) iron oxide particles. When put into contact with a blood sample of approximately $30 \mu \mathrm{L}$, ecarin triggers a coagulation reaction by conversion of prothrombin into meizothrombin, which subsequently polymerizes fibrinogen into fibrin eventually leading to the formation of a stable clot. ${ }^{8}$ An oscillating magnetic field under the card produced by the analyzer causes the iron particles to move constantly. The increasing restriction of movement caused by the forming fibrin clot is detected by an infrared optical system and reported as a clotting time. The presence of a thrombin inhibitor extends the clotting time proportionately to its concentration in the blood sample.

\section{Blinding}

All POC device operators were blinded to the results of all other coagulation assays as well as those of UPLC-MS/MS. External technicians conducting UPLC-MS/MS were blinded to the results of all other coagulation assays (including POCECT) as well as patient number and sampling time point. External technicians conducting laboratory-based ECT were blinded to the results of all other coagulation assays and those of UPLC-MS/MS as well as patient number and sampling time point. Fully automated laboratory-based measurements (including BDTI) were conducted during routine operation at our central laboratory where technicians were blinded to the results of POC-ECT as well as UPLC-MS/MS, and laboratory-based ECT.

\section{Calibration and Limit of Detection}

To assess the feasibility and accuracy of calibrated POC-ECT measurements of dabigatran plasma concentrations, we retrospectively analyzed 138 rethawed $\mathrm{CP}$ samples from two previous studies. ${ }^{4,5}$ POC-ECT values were correlated with actual dabigatran concentrations determined by UPLC-MS/MS to yield a regression line, which was used for calculation of calibrated POC-ECT from CP in the prospective study.

The limit of detection (LOD) was defined using POC-ECT values gained from the baseline measurements of those patients who had not taken any dabigatran prior to any blood samples. The estimated plasma concentration of these samples was determined using the calibration function. Mean and standard deviation of the results were calculated. The LOD was determined by adding three standard deviations to the mean value. For further analyses, calibrated values below the LOD were defined as $0 \mathrm{ng} / \mathrm{mL}$.

\section{Interassay Variability, Interaction with Heparins and Direct Oral Xa-Inhibitors, and Monitoring of Dabigatran Reversal Using Idarucizumab}

In addition to the abovementioned experiments, we performed in vitro experiments to determine interassay variability, interaction of UFH, LMWH, and direct oral Xa-inhibitors with POC-ECT, and feasibility of drug reversal monitoring (for details please see - Supplementary Methods in the -Supplementary Material [available in the online version]).

\section{Statistical Analyses}

Pearson's correlation coefficient was used to quantify the strength of correlation between coagulation assay test results and UPLC-MS/MS. Diagnostic accuracy of coagulation assays regarding detection of clinically relevant dabigatran plasma concentrations (i.e., $>30$ and $>50 \mathrm{ng} / \mathrm{mL})^{9-12}$ is expressed in terms of sensitivity, specificity, positive and negative predictive value as well as likelihood ratio including respective $95 \%$ confidence intervals. Contrary to prior publications from our group, ${ }^{4,5}$ sensitivity is defined as the percentage of samples containing clinically relevant dabigatran plasma concentrations that were correctly identified by the respective coagulation assay and thus as theoretically belonging to a patient ineligible for thrombolysis/surgery or requiring reversal therapy. Specificity is defined as the percentage of samples containing dabigatran concentrations $\leq 30 \mathrm{ng} / \mathrm{mL}$ that were correctly identified by the respective coagulation assay. Positive predictive value is defined as the percentage of samples with dabigatran concentrations $>30$ $\mathrm{ng} / \mathrm{mL}$ of all samples identified as containing relevant drug levels by the respective coagulation assay and negative predictive value is defined as the percentage of samples with dabigatran concentrations $\leq 30 \mathrm{ng} / \mathrm{mL}$ of all samples identified as containing no relevant drug levels by the respective coagulation assays. Receiver operating characteristic (ROC) curves were drawn and the area under the ROC curve (AUROC) was calculated for each coagulation assay. The ideal cut-off point was defined for each coagulation assay to yield a target sensitivity of at least $95 \%$-predefined as sufficiently safe for clinical application-and the highest possible specificity to avoid false-negative results but simultaneously to identify the largest number of patients eligible for emergency treatment such as thrombolysis or emergency surgery. 
The 95\% confidence intervals for all proportions were calculated according to the efficient-score method as described by Newcombe ${ }^{13}$ using the free online VassarStats Clinical Calculator $1 .{ }^{14}$ AUROCs were compared using the VassarStats Clinical Calculator according to suggestions made by Hanley and McNeil. ${ }^{15,16}$ SPSS version 24 (IBM, Armonk, New York, United States) was used for all other statistical analyses as well as visualization of scatter and Bland-Altman plots and ROC curves. Bland-Altman plots were modified following suggestions made by Krouwer. ${ }^{17}$

Using a two-tailed approach, an $\alpha$-level of $<0.05$ was considered statistically significant.

This study was performed in accordance with the STARD (Standards for Reporting Diagnostic Accuracy) guidelines for studies on diagnostic tests. ${ }^{18}$

\section{Results}

\section{Clinical Study}

Between September 2015 and April 2016, 42 patients gave written informed consent to study participation. A total of 40 were enrolled in the study; 20 receiving first dose of dabigatran and 20 already on dabigatran treatment. Baseline demographics are summarized in -Table $\mathbf{1}$ and baseline laboratory values are summarized in -Supplementary Table S1 (available in the online version). Two patients were screened but did not meet eligibility criteria.

A total of 240 blood samples were collected and analyzed as described above. According to UPLC-MS/MS, they contained 0 to $275 \mathrm{ng} / \mathrm{mL}$ of dabigatran. Using $\mathrm{WB} / \mathrm{CB} / \mathrm{CP}$, POCECT ranged from 20 to $186 / 184 / 316$ seconds. Calibrated laboratory-based ECT yielded estimated dabigatran concentrations of 0 to $265 \mathrm{ng} / \mathrm{mL}$ and calibrated laboratory-based BDTI assay concentrations of 0 to $298 \mathrm{ng} / \mathrm{mL}$. No samples were lost to analysis.

\section{Correlation and Receiver Operating Characteristics}

POC-ECT values showed a strong linear correlation with dabigatran plasma concentrations as determined by UPLC-MS/MS (see -Fig. $\mathbf{1}$ for illustration of linear regression).

Correlation was highest for POC-ECT with CP $\left(R^{2}=0.92\right.$, $p<0.001 ; \quad$ - Fig. 1C) followed by CB $\left(R^{2}=0.90\right.$, $p<0.001$; -Fig. 1B $)$ and $\mathrm{WB}\left(R^{2}=0.78, p<0.001\right.$; - Fig. 1A). Full results of ROC analyses are summarized in - Tables 2 and 3 and $\boldsymbol{\sim}$ Supplementary Fig. $\mathbf{S 1}$ (available in the online version).

\section{Calibration and Limit of Detection}

Frozen/rethawed CP samples used for retrospective POC-ECT measurements contained 0 to $371 \mathrm{ng} / \mathrm{mL}$ of dabigatran. POCECT values ranged from 20 to 219 seconds. Linear regression analysis revealed a strong correlation of POC-ECT with dabigatran plasma concentrations $\left(R^{2}=0.91\right)$. The calibration function was determined as: estimated dabigatran plasma concentration $(\mathrm{ng} / \mathrm{mL})=1.521 *$ POC-ECT from CP (s) 40.369. Applying the calibration function to dabigatranfree baseline measurements from the prospective study, the LOD was determined to be $9 \mathrm{ng} / \mathrm{mL}$.
Table 1 Patient baseline characteristics $(N=40)$

\begin{tabular}{|c|c|}
\hline Age & $67 \pm 14 y$ \\
\hline Female sex & $19(47.5 \%)$ \\
\hline Dabigatran dose & $\begin{array}{l}150 \mathrm{mg} \text { BD: } 26(65 \%) \\
110 \mathrm{mg} \text { BD: } 14(35 \%)\end{array}$ \\
\hline Body weight & $78 \pm 16.5 \mathrm{~kg}$ \\
\hline Body mass index & $27 \pm 4.3 \mathrm{~kg} / \mathrm{m}^{2}$ \\
\hline \multicolumn{2}{|l|}{ Risk factors } \\
\hline Arterial hypertension & 27 (68\%) \\
\hline Hyperlipidemia & $20(50 \%)$ \\
\hline Diabetes mellitus & $10(25 \%)$ \\
\hline History of stroke & $26(65 \%)$ \\
\hline Congestive heart failure & $8(20 \%)$ \\
\hline $\begin{array}{l}\text { Coronary heart disease } \\
\text { or history of myocardial } \\
\text { infarction }\end{array}$ & $4(10 \%)$ \\
\hline Smoking & $2(5 \%)$ \\
\hline Alcohol abuse & $2(5 \%)$ \\
\hline \multicolumn{2}{|l|}{ Indication for dabigatran therapy } \\
\hline Atrial fibrillation & $19(48 \%)$ \\
\hline AF ablation & $6(15 \%)$ \\
\hline $\begin{array}{l}\text { Stroke associated with patent } \\
\text { foramen ovale }\end{array}$ & $13(33 \%)$ \\
\hline $\begin{array}{l}\text { Repeated embolic stroke of } \\
\text { undetermined source }\end{array}$ & $1(3 \%)$ \\
\hline Deep vein thrombosis & $1(3 \%)$ \\
\hline \multicolumn{2}{|l|}{ Concomitant antiplatelet therapy } \\
\hline Acetylsalicylic acid & $22(55 \%)$ \\
\hline Clopidogrel & $1(3 \%)$ \\
\hline
\end{tabular}

Abbreviations: AF, atrial fibrillation; $\mathrm{BD}$, twice a day. Note: Continuous variables are displayed as mean \pm standard deviation. Nominal variables are displayed as absolute quantity (percentage).

\section{Agreement of Calibrated POC-ECT and UPLC-MS/MS}

Agreement of calibrated POC-ECT with CP and calibrated laboratory-based ECT and BDTI with UPLC-MS/MS is visualized using Bland-Altman plots and mean, as well as standard deviation of the differences between calibrated and massspectrometric dabigatran concentrations was calculated both for the whole dataset and for a low concentration range of 0 to $100 \mathrm{ng} / \mathrm{mL}$ (-Fig. 2).

Bias of calibrated laboratory-based ECT was negligible overall and in the low concentration range. Bias of calibrated POC-ECT with $\mathrm{CP}$ was negligible in the low-concentration range and slightly positive $(8.5 \mathrm{ng} / \mathrm{mL})$ overall with a trend toward overestimation of true dabigatran plasma concentrations at higher levels. Calibrated laboratory-based BDTI showed a negative bias overall $(-11.4 \mathrm{ng} / \mathrm{mL})$ and, more importantly, in the low range $(-12.1 \mathrm{ng} / \mathrm{mL})$. The standard deviation of calibrated POC-ECT values from the true dabigatran plasma concentration range was found to be higher than that of laboratory-based ECT and comparable to that of laboratory-based BDTI. 

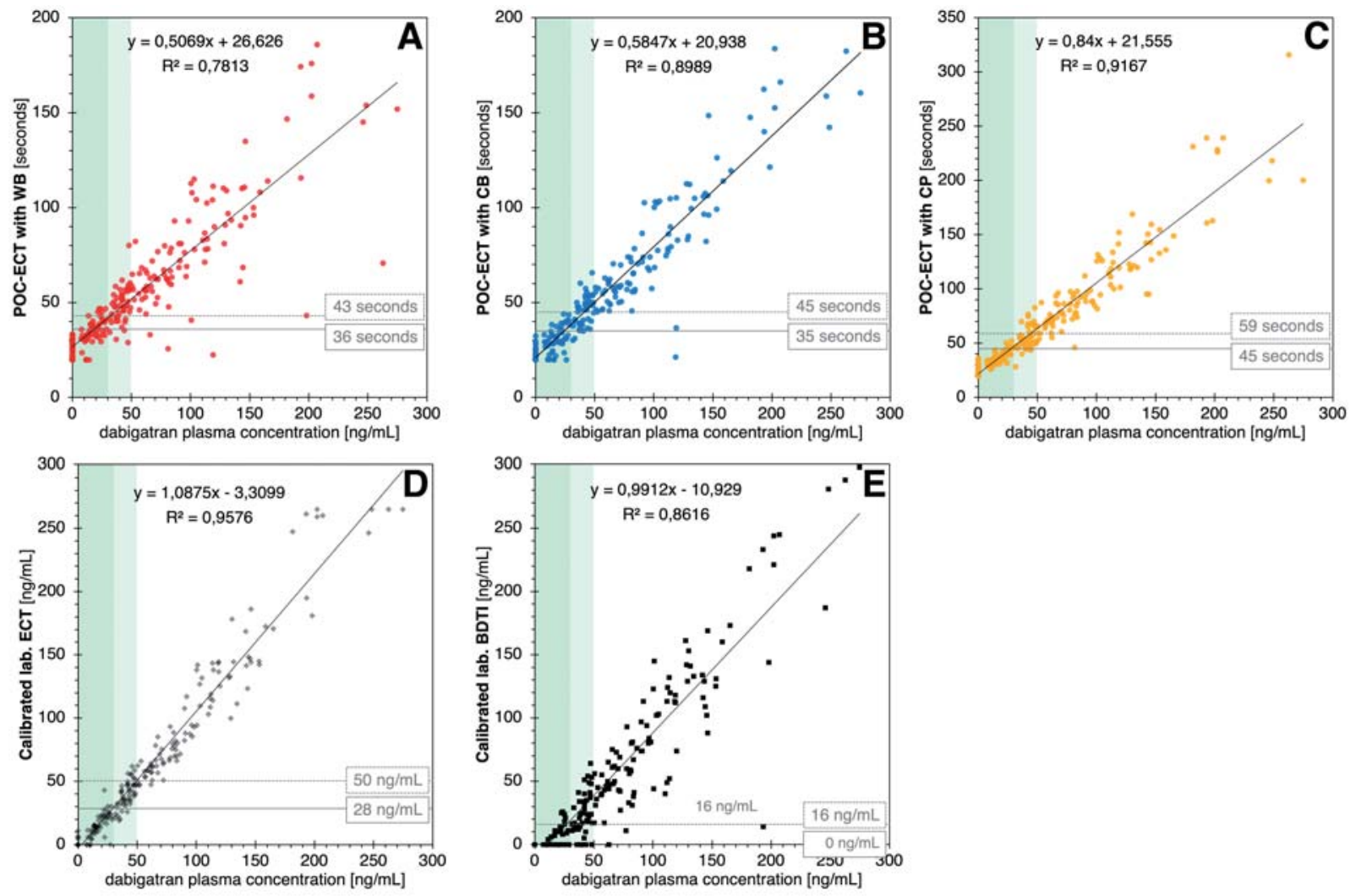

Fig. 1 Diagnostic accuracy of point-of-care ecarin clotting time (POC-ECT), laboratory-based ECT, and laboratory-based Biophen Direct Thrombin Inhibitor (BDTI) assay. Scatter plots illustrate the correlation between dabigatran plasma concentrations determined by ultraperformance liquid chromatography/tandem mass spectrometry and (A) POC-ECT with whole blood (WB), (B) citrated blood (CB), (C) citrated plasma (CP) as well as (D) calibrated laboratory-based (lab.) ECT, and (E) calibrated laboratory-based (lab) BDTI. Green shaded bars indicate the treatment-relevant dabigatran plasma concentration thresholds of 30 (darker) and $50 \mathrm{ng} / \mathrm{mL}$ (lighter). Horizontal gray lines indicate suggested optimal cut-offs providing $>95 \%$ sensitivity for detection of samples containing $>30$ (solid) and $>50 \mathrm{ng} / \mathrm{mL}$ (dashed) of dabigatran. Diagonal black lines indicate regression lines with their respective equations and the squared Pearson's correlation coefficient $\left(R^{2}\right)$ to be found in the upper left corner of the diagrams.

\section{Interassay Variability, Interaction with Heparins, Direct Oral Xa-Inhibitors and, Idarucizumab}

Assessment of interassay variability yielded a coefficient of variance of $12.3 \%$ for normal plasma containing no dabigatran, $8.7 \%$ at $30 \mathrm{ng} / \mathrm{mL}$, and $11.5 \%$ at $255 \mathrm{ng} / \mathrm{mL}$ for calibrator plasma, and $8.1 \%$ at $100.6 \mathrm{ng} / \mathrm{mL}$ and $14.7 \%$ at $198.1 \mathrm{ng} / \mathrm{mL}$ for rethawed patient $\mathrm{CP}$ samples. Artificial addition of therapeutic and supratherapeutic doses of UFH, LMWH, or direct oral Xa-inhibitors did not alter the POC-ECT results (see -Supplementary Tables $\mathbf{5 2}$ and \$3, available in the online version). POC-ECT results accurately reflected idarucizumabinduced reversal of anticoagulation. Plasma samples containing dabigatran bound to idarucizumab yielded normal POC-ECT values (see - Supplementary Table S4, available in the online version).

\section{Discussion}

This study represents the first clinical study to qualitatively and quantitatively evaluate a DOAC-specific POC coagulation assay.

Overall, POC-ECT using WB, CB, and $\mathrm{CP}$ is feasible and testing with all three different sample types yielded POC-ECT values that strongly correlate with actual dabigatran plasma concentrations as determined by UPLC-MS/MS (gold stan- dard for dabigatran concentration measurement) with little scattering and thus high accuracy in the clinically most relevant concentration range around the current thresholds for thrombolysis, reversal therapy, and emergency surgery. ${ }^{9-12}$

POC-ECT using WB and CB was performed under routine conditions at the bedside and without relevant loss of time between sampling and testing. Regarding detection of dabigatran plasma concentrations $>30$ and $>50 \mathrm{ng} / \mathrm{mL}$, accuracy of POC-ECT with CB is comparable to that of laboratory-based $\mathrm{ECT}$, which in our case was measured in batch under stable conditions. Laboratory-based BDTI, on the other hand, did not reach this level of performance.

For technical reasons, POC-ECT with WB is performed minimally faster than with $\mathrm{CB}$. Due to the limited stability of noncitrated, nonheparinized WB in a plastic syringe, on the other hand, test performance may suffer (as reflected in poorer ROCs).

Accuracy of POC-ECT using CP may be comparable or even superior to POC-ECT with CB. Due to acquisition and handling, however, use of $\mathrm{CP}$ is complicated and comes with significant loss of valuable time. $C B$ thus seems to represent the most suitable sample type for emergency POC-ECT.

The agreement between calibrated POC-ECT and actual dabigatran plasma concentrations shows that quantitative 
Table 2 Sensitivity and specificity regarding detection of dabigatran concentrations $>30$ and $>50 \mathrm{ng} / \mathrm{mL}$

\begin{tabular}{|c|c|c|c|c|c|c|c|}
\hline Test & $\begin{array}{l}\text { Threshold } \\
\text { (ng/mL) }\end{array}$ & $\begin{array}{l}\text { (Ideal) } \\
\text { cut-off }\end{array}$ & Sensitivity (\%) & Specificity (\%) & LR & PPV (\%) & NPV (\%) \\
\hline \multirow{2}{*}{$\begin{array}{l}\text { POC-ECT } \\
\text { WB }\end{array}$} & $>30$ & $36 \mathrm{~s}$ & 95.1 [89.8-97.8] & $81.4[72.0-88.3]$ & $5.13[3.37-7.89]$ & 88.3 [81.9-92.7] & 91.9 [83.4-96.4] \\
\hline & $>50$ & $43 \mathrm{~s}$ & 95.8 [89.1-98.7] & $73.6[65.5-80.4]$ & $3.63[2.76-4.79]$ & $70.8[62.0-78.2]$ & 96.4 [90.4-98.8] \\
\hline \multirow{2}{*}{$\begin{array}{l}\text { POC-ECT } \\
\text { CB }\end{array}$} & $>30$ & $35 s$ & 97.2 [92.5-99.1] & $86.6[77.8-92.4]$ & $7.25[4.37-12.04]$ & $91.4[85.5-95.2]$ & 95.5 [88.1-98.5] \\
\hline & $>50$ & $45 s$ & 97.9 [92.0-99.6] & $60.4[51.9-68.4]$ & $2.47[2.02-3.03]$ & $62.3[54.0-69.9]$ & 97.8 [91.4-99.6] \\
\hline \multirow{2}{*}{$\begin{array}{l}\text { POC-ECT } \\
\text { CP }\end{array}$} & $>30$ & $45 s$ & 96.5 [91.6-98.7] & 93.8 [86.5-97.5] & $15.60[7.18-33.89]$ & $95.8[90.8-98.3]$ & 94.8 [87.7-98.1] \\
\hline & $>50$ & $59 \mathrm{~s}$ & 96.9 [90.5-99.2] & 91.0 [84.8-94.9] & $10.73[6.38-18.04]$ & 87.7 [79.6-93.0] & 97.8 [93.1-99.4] \\
\hline \multirow{3}{*}{$\begin{array}{l}\text { Calibrated } \\
\text { POC-ECT }\end{array}$} & $>30$ & $28 \mathrm{ng} / \mathrm{mL}$ & 96.5 [91.6-98.7] & 93.8 [86.5-97.5] & $15.60[7.18-33.89]$ & $95.8[90.8-98.3]$ & 94.8 [87.7-98.1] \\
\hline & & $30 \mathrm{ng} / \mathrm{mL}^{\mathrm{a}}$ & 92.3 [86.3-95.9] & 95.9 [89.2-98.7] & $22.38[8.56-58.50]$ & $97.1[92.2-99.1]$ & 89.4 [81.5-94.3] \\
\hline & $>50$ & $50 \mathrm{ng} / \mathrm{mL}$ & 95.8 [89.1-98.7] & $90.1[84.8-94.9]$ & $10.62[6.31-17.86]$ & $87.6[79.4-93.0]$ & 97.0 [92.1-99.0] \\
\hline \multirow{3}{*}{$\begin{array}{l}\text { Calibrated } \\
\text { lab. ECT }\end{array}$} & $>30$ & $28 \mathrm{ng} / \mathrm{mL}$ & $95.1[89.8-97.8]$ & 96.9 [90.6-99.2] & 30.75 [10.09-93.74] & $97.8[93.3-99.4]$ & 93.1 [85.8-96.9] \\
\hline & & $30 \mathrm{ng} / \mathrm{mL}^{\mathrm{a}}$ & 94.4 [88.9-97.3] & 96.9 [90.6-99.2] & 30.52 [10.01-93.05] & $97.8[93.3-99.4]$ & 92.2 [84.7-96.3] \\
\hline & $>50$ & $50 \mathrm{ng} / \mathrm{mL}$ & 96.9 [90.5-99.2] & 70.1 [61.9-77.3] & $3.24[2.52-4.18]$ & $68.4[59.8-75.9]$ & 97.1 [91.2-99.3] \\
\hline \multirow{4}{*}{$\begin{array}{l}\text { Calibrated } \\
\text { lab. BDTI }\end{array}$} & $>30$ & $0 \mathrm{ng} / \mathrm{mL}$ & $95.1[89.8-97.8]$ & $82.5[73.1-89.2]$ & $5.43[3.52-8.37]$ & $88.9[82.5-93.2]$ & $92.0[83.6-96.4]$ \\
\hline & & $30 \mathrm{ng} / \mathrm{mL}^{\mathrm{a}}$ & $74.1[66.0-80.9]$ & $100[95.3-100]$ & - & $100[95.6-100]$ & 72.4 [63.9-79.6] \\
\hline & $>50$ & $16 \mathrm{ng} / \mathrm{mL}$ & 96.9 [90.5-99.2] & $75.7[67.7-82.3]$ & $3.99[2.98-5.33]$ & $72.7[63.9-80.0]$ & 97.3 [91.8-99.3] \\
\hline & & $50 \mathrm{ng} / \mathrm{mL}^{\mathrm{a}}$ & 69.8 [59.4-78.5] & $97.2[92.6-99.1]$ & $25.13[9.48-66.62]$ & $94.4[85.5-98.2]$ & $82.8[76.1-88.0]$ \\
\hline
\end{tabular}

Abbreviations: BDTI, Biophen Direct Thrombin Inhibitor assay; CB, citrated blood; CP, citrated plasma; ECT, ecarin clotting time; lab., laboratorybased; LR, likelihood ratio; NPV, negative predictive value; POC-ECT, point-of-care ecarin clotting time; PPV, positive predictive value; WB, whole blood.

Note: The ideal cut-off point (in bold letters) was defined for each coagulation assay as the lowest test result yielding a target sensitivity of at least $95 \%$ regarding detection of dabigatran concentrations $>30$ and $>50 \mathrm{ng} / \mathrm{mL}$.

aFor calibrated assays, all values were calculated for test results of " $30 \mathrm{ng} / \mathrm{mL}$ " and " $50 \mathrm{ng} / \mathrm{mL}$ " in addition to the ideal cut-off. Sensitivity, specificity, PPV, and NPV are displayed in \% with 95\% confidence intervals in square brackets. LR is displayed with $95 \%$ confidence intervals in square brackets.

Table 3 Comparison of areas under the ROC curves for detection of dabigatran plasma levels $>30$ and $>50 \mathrm{ng} / \mathrm{mL}$

\begin{tabular}{|l|l|l|l|}
\hline Method & Threshold $(\mathbf{n g} / \mathbf{m L})$ & AUROC & Two-tailed $p$-Value \\
\hline \multirow{3}{*}{ Lab. ECT } & $>30$ & $0.99[0.99-1.00]$ & Reference \\
\cline { 2 - 4 } & $>50$ & $0.99[0.99-1.00]$ & Reference \\
\hline \multirow{3}{*}{ Lab. BDTI } & $>30$ & $0.96[0.94-0.98]$ & $0.011^{\mathrm{a}}$ \\
\cline { 2 - 4 } & $>50$ & $0.97[0.95-0.99]$ & 0.052 \\
\hline \multirow{2}{*}{ POC-ECT with WB } & $>30$ & $0.97[0.95-0.99]$ & $0.028^{\mathrm{a}}$ \\
\cline { 2 - 4 } & $>50$ & $0.95[0.92-0.98]$ & $0.011^{\mathrm{a}}$ \\
\hline \multirow{2}{*}{ POC-ECT with CB } & $>30$ & $0.98[0.97-1.00]$ & 0.215 \\
\cline { 2 - 4 } & $>50$ & $0.97[0.95-0.99]$ & 0.058 \\
\hline \multirow{2}{*}{ POC-ECT with CP } & $>30$ & $0.99[0.98-1.00]$ & 0.700 \\
\cline { 2 - 4 } & $>50$ & $0.98[0.97-1.00]$ & 0.351 \\
\hline
\end{tabular}

Abbreviations: AUROC, area under the receiver operating curve; BDTI, Biophen Direct Thrombin Inhibitor assay; CB, citrated blood; CP, citrated plasma; ECT, ecarin clotting time; POC-ECT, point-of-care ecarin clotting time; ROC, receiver operating curve; WB, whole blood.

Note: This table lists the AUROCs found in - Supplementary Fig. S1 (available in the online version). We compared AUROCs of all test modalities, using calibrated laboratory-based ECT (lab. ECT), which was performed in batch under controlled conditions, as the reference. At the $30 \mathrm{ng} / \mathrm{mL}$ threshold, we determined the AUROC of POC-ECT with WB and that of the calibrated laboratory-based BDTI (lab. BDTI) to be significantly smaller while AUROC of POC-ECT with CB as well as CP did not differ significantly from the reference. For the $50 \mathrm{ng} / \mathrm{mL}$ threshold, only POC-ECT with WB performed significantly worse than lab. ECT. AUROC is displayed with $95 \%$ confidence intervals in square brackets.

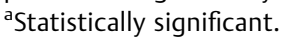

estimation of drug levels is possible. However, this is currently only true for $\mathrm{CP}$.

Due to the delay associated with the creation of plasma, we would encourage the use of raw POC-ECT performed ideally with $\mathrm{CB}$ (or $\mathrm{WB})$, and a sufficiently low cut-off (-Table 2) to rule out the presence of clinically relevant dabigatran plasma concentrations in patients who have not recently received any other anticoagulants influencing IIa activity (except UFH), e.g., VKA, hirudins.

If calibrated assays are used for (emergency) decision making, test results given in "ng/mL" cannot necessarily be taken literally and may involve significant imprecision, as 

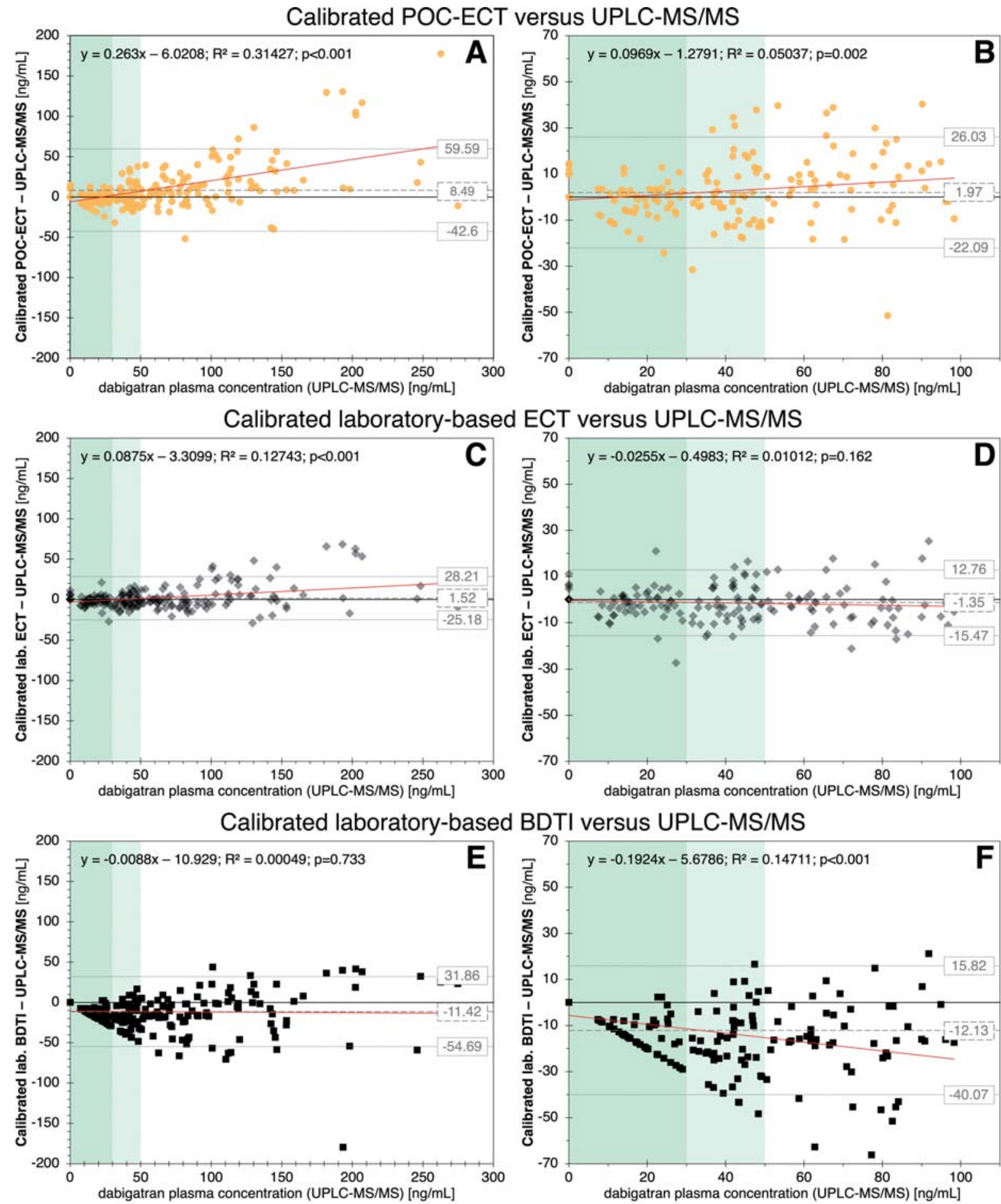

Fig. 2 Comparison of agreement with ultra-performance liquid chromatography/tandem mass spectrometry (UPLC-MS/MS) between calibrated point-of-care ecarin clotting time (POC-ECT) and two calibrated laboratory-based anti-lla assays. Bland-Altman plots are used to display the agreement level between UPLC-MS/MS and calibrated POC-ECT: (A) all data and (B) $0-100 \mathrm{ng} / \mathrm{mL}$; calibrated laboratory-based (lab.) ECT: (C) all data and (D) 0-100 ng/mL; as well as calibrated laboratory-based (lab.) Biophen Direct Thrombin Inhibitor (BDTI) assay: (E) all data and (F) $0-100 \mathrm{ng} / \mathrm{mL}$; all measurements were performed using citrated plasma. Gray horizontal lines indicate a distance of 1.96 standard deviations (short-dashed) from the mean (long-dashed). Green shaded bars indicate the dabigatran plasma concentration treatment-relevant thresholds of 30 and $50 \mathrm{ng} / \mathrm{mL}$. Diagonal red lines indicate regression curves with their respective equations and the squared Pearson's correlation coefficient $\left(R^{2}\right)$ to be found in the upper left corner of the diagrams.

shown for the laboratory-based BDTI: despite its high correlation with UPLC-MS/MS, we found safe cut-off values (i.e., detection of dabigatran concentrations $>30$ or $>50 \mathrm{ng} / \mathrm{mL}$ with $>95 \%$ sensitivity) only below the test's LOD $(0 \mathrm{ng} / \mathrm{mL})$ or at "16 ng/mL," respectively. In contrast, for calibrated POCECT with $\mathrm{CP}$ as well as calibrated laboratory-based ECT, the ideal cut-off values were either close $(28 \mathrm{ng} / \mathrm{mL})$ or equal $(50 \mathrm{ng} / \mathrm{mL}$ ) to the real thresholds (-Table 2 ). 


\section{Strengths and Limitations}

POC measurements were performed at the bedside under reallife conditions. Patients are well characterized and no artificial spiking of samples was performed for the main analyses. Within our dataset, low to moderate dabigatran concentrations are well represented, supporting the validity of our ROC analyses around the treatment-relevant thresholds of 30 and $50 \mathrm{ng} / \mathrm{mL}$. The sampling time points were chosen on the basis of positive experience in earlier studies of our work group, where we were able to collect samples containing a wide range of dabigatran plasma concentrations. ${ }^{4,5}$

When comparing our results to data from unspecific global coagulation POC published by our group, ${ }^{4,5}$ diagnostic performance in emergency decision making is significantly improved through the use of POC-ECT.

A limitation of our study is that patients using other anticoagulants were excluded. In real-life emergency situations, however, a detailed medical history of the patient might be unavailable. To address this issue and assess the anti-IIa specificity of the assay, we conducted measurements with samples containing dabigatran as well as UFH, LMWH, and the currently approved direct oral Xa-inhibitors (apixaban, edoxaban, and rivaroxaban) in high concentrations, which revealed no significant interaction of the substances with POC-ECT.

The high anti-Ila specificity of POC-ECT assures accurate detection of dabigatran in blood samples of a dabigatrantreated patient. Intake of other anticoagulants, however, will still have to be excluded by the patient's history or other coagulation assays. Unfortunately, there is currently no antiXa-specific POC assay available.

Despite the potential use of POC-ECT in the emergency department, we did not include any patients in the emergency setting. The nonemergency setting was chosen to ensure fast patient recruitment and feasibility of multiple POC measurements per patient allowing for the collection of baseline blood samples as well as samples containing a wide spectrum of dabigatran plasma concentrations.

The ideal cut-offs found in our dataset were defined retrospectively and do not necessarily translate to other batches of POC-ECT test cards. To enable the safe use of POC-ECT (with CB or WB) as an emergency diagnostic tool in the future, the manufacturer will either have to provide respective POC-ECT cut-offs for the (current) treatmentrelevant dabigatran plasma concentration thresholds or offer a CB- or WB-based calibrated POC-ECT.

\section{POC-ECT in the Context of Acute Stroke Care}

Given the high diagnostic accuracy of the DTM test card-based POC-ECT in detecting relevant plasma concentrations of dabigatran, we deem it suitable to be integrated in the acute stroke care work flow, where POC-INR has already become the diagnostic standard in VKA-treated patients. Pivotal features of the device and assay are the portability of the battery powered Cascade Abrazo POC device, the possible use of WB (noncitrated or citrated) without the need for centrifugation, and the fast availability of test results as longer intervals would affect admission-to-treatment times.
At this moment in time, other POC systems are less suited for this task: rotational thromboelastometry (ROTEM) and thromboelastography (TEG) have also been evaluated for POC coagulation assessment in DOAC-treated patients as well as monitoring of dabigatran reversal with idarucizumab (in vitro and in a porcine polytrauma model). ${ }^{19-22}$ The size of respective devices, however, limits transportability and the duration of measurements is much longer compared with the POC-ECT method. Furthermore, sensitivity to low plasma concentrations, which are relevant for guiding emergency decision making, has generally been reported as being low, at least if the reagents used are not DOAC-specific. Akman and colleagues have suggested that dabigatran reversal using idarucizumab can be monitored using ROTEM and TEG, 22 while a study by Takeshita and colleagues indicated that incomplete reversal of dabigatran might not be accurately reflected by ROTEM. ${ }^{21}$ One recent evaluation of a ROTEM method using a thrombin trigger showed promising results regarding speed of measurements and correlation between clotting times and dabigatran plasma concentrations in an in vitro study. The data acquired in a small clinical substudy, however, were only analyzed qualitatively and for a threshold, which is not regarded as relevant for clinical decision making. ${ }^{23}$ A thorough prospective quantitative and quantitative clinical evaluation of ROTEM/TEG-based POC coagulation testing in DOAC-treated patients, including clinically relevant safe-for-treatment thresholds, is-to the best of our knowledge-still lacking.

A completely different approach is taken by a recently developed urine test, which is designed to provide information about the presence or absence of a urine concentration of dabigatran or FXa inhibitor above $95 \mathrm{ng} / \mathrm{mL}$. The advantage of this assay is that it will detect both substance classes simultaneously. The duration of this merely qualitative measurement, however, is also more than 10 minutes and it naturally does not allow for determination of drug plasma concentration and consequently the level of anticoagulation. ${ }^{24}$

Previous publications of our group have shown that determination of DOAC plasma concentrations (including dabigatran) is possible within certain limits. As a linear correlation between POC test results and actual DOAC plasma concentrations is not sufficient for qualitative estimation, POC testing using global coagulation assays is only able to provide qualitative information about the presence of DOAC, however, with high accuracy for predefined clinically relevant thresholds. ${ }^{4-6}$ Using the suggested decision model, a relevant percentage of patients who may receive intravenous thrombolysis (or in which reversal therapy is unnecessary) is safely identified.

The Cascade Abrazo currently lacks an anti-FXa specific coagulation assay. The so-called ENOX test card is currently under evaluation (NCT02825394).

\section{Conclusion}

This study represents the first qualitative and quantitative evaluation of DOAC-specific POC coagulation testing in dabigatran-treated patients. Correlation of POC with UPLC-MS/MS results is excellent and-through calibration of plasma-based 
measurements-allows for quantitative estimation of actual dabigatran plasma concentrations.

POC-ECT is suitable for distinguishing between dabigatran concentrations of above and below 30 and $50 \mathrm{ng} / \mathrm{mL}$, potentially enabling clinicians to reliably detect or exclude the presence of relevant drug levels in the emergency setting without unnecessary loss of time. POC-ECT is not influenced by UFH, LMWH, or direct oral Xa-inhibitors and accurately reflects normalization of coagulation following dabigatran reversal with idarucizumab.

\section{What is known about this topic?}

- In stroke patients or prior emergency surgery, rapid assessment of coagulation status is necessary to guide thrombolysis or reversal of anticoagulation.

- Available point-of-care global coagulation assays do only provide (limited) qualitative assessment, but do not allow for quantitative coagulation testing in patients treated with direct oral anticoagulants including the thrombin inhibitor dabigatran.

\section{What does this paper add?}

- This is the first study evaluating point-of-care ecarin clotting time (POC-ECT).

- POC-ECT accurately reflected dabigatran plasma concentrations and is not influenced by other anticoagulants.

- POC-ECT could improve clinical care of dabigatrantreated patients by making fast and precise assessment of coagulation available to emergency physician.

\section{Conflict of Interest}

F.H. reports nonfinancial support from Helena Laboratories, during the conduct of the study; other from Bayer, other from Daiichi Sankyo, outside the submitted work. I. B. reports personal fees and other from Bristol-Myers Squibb/Pfizer, grants and personal fees from Siemens Healthcare, personal fees from CSL Behring, other from Aspen, other from LFB Biomedicaments, outside the submitted work. H.R. reports nonfinancial support from Helena Laboratories, during the conduct of the study. U. Z. reports nonfinancial support from Helena Laboratories, during the conduct of the study; grants and personal fees from Biogen Idec, personal fees from Bayer Vital, personal fees from Bristol-Myers Squibb/Pfizer, personal fees from CorTec, personal fees from Medtronic, grants from Servier, grants from Janssen Pharmaceutica NV, outside the submitted work. S.P. reports nonfinancial support from Helena Laboratories, during the conduct of the study; personal fees from Bayer, grants and personal fees from Boehringer-Ingelheim, grants and personal fees from Bristol-Myers Squibb/Pfizer, grants and personal fees from Daiichi Sankyo, personal fees from Werfen, outside the submitted work.

\section{Acknowledgments}

This work was supported by Helena Laboratories (Beaumont, Texas, United States) which provided Abrazo test devices and cards free of charge. No further financial/material support was received. Helena Laboratories had no influence on/was not involved in study design, collection, analysis, interpretation of data, writing of the report, or in the decision to submit the manuscript for publication.

We thank Louise Härtig for language revision of the manuscript.

\section{References}

1 Walter S, Kostopoulos P, Haass A, et al. Point-of-care laboratory halves door-to-therapy-decision time in acute stroke. Ann Neurol 2011;69(03):581-586

2 Rizos T, Herweh C, Jenetzky E, et al. Point-of-care international normalized ratio testing accelerates thrombolysis in patients with acute ischemic stroke using oral anticoagulants. Stroke 2009;40 (11):3547-3551

3 Zantek ND, Hayward CP, Simcox TG, Smock KJ, Hsu P, Van Cott EM. An assessment of the state of current practice in coagulation laboratories. Am J Clin Pathol 2016;146(03):378-383

4 Ebner M, Peter A, Spencer C, et al. Point-of-care testing of coagulation in patients treated with non-vitamin $\mathrm{K}$ antagonist oral anticoagulants. Stroke 2015;46(10):2741-2747

5 Ebner M, Birschmann I, Peter A, et al. Point-of-care testing for emergency assessment of coagulation in patients treated with direct oral anticoagulants. Crit Care 2017;21(01):32

6 Härtig F, Birschmann I, Peter A, et al. Point-of-care testing of coagulation in patients treated with edoxaban. J Thromb Thrombolysis 2020;50(03):632-639

7 Kuhn J, Gripp T, Flieder T, et al. UPLC-MRM mass spectrometry method for measurement of the coagulation inhibitors dabigatran and rivaroxaban in human plasma and its comparison with functional assays. PLoS One 2015;10(12):e0145478

8 Novoa E, Seegers WH. Mechanisms of alpha-thrombin and betathrombin-E formation: use of ecarin for isolation of meizothrombin 1. Thromb Res 1980;18(05):657-668

9 Ahmed N, Steiner T, Caso V, Wahlgren NESO-KSU session participants. Recommendations from the ESO-Karolinska Stroke Update Conference, Stockholm 13-15 November 2016. Eur Stroke J 2017;2(02):95-102

10 Touzé E, Gruel Y, Gouin-Thibault I, et al. Intravenous thrombolysis for acute ischaemic stroke in patients on direct oral anticoagulants. Eur J Neurol 2018;25(05):747-e52

11 Pernod G, Albaladejo P, Godier A, et al;Working Group on Perioperative Haemostasis. Management of major bleeding complications and emergency surgery in patients on long-term treatment with direct oral anticoagulants, thrombin or factor-Xa inhibitors: proposals of the working group on perioperative haemostasis (GIHP) - March 2013. Arch Cardiovasc Dis 2013;106 (6-7):382-393

12 Levy JH, Ageno W, Chan NC, Crowther M, Verhamme P, Weitz JISubcommittee on Control of Anticoagulation. When and how to use antidotes for the reversal of direct oral anticoagulants: guidance from the SSC of the ISTH. J Thromb Haemost 2016;14 (03):623-627

13 Newcombe RG. Two-sided confidence intervals for the single proportion: comparison of seven methods. Stat Med 1998;17 (08):857-872

14 Steiner T, Freiberger A, Griebe M, et al. International normalised ratio normalisation in patients with coumarin-related intracranial haemorrhages-the INCH trial: a randomised controlled multicentre trial to compare safety and preliminary efficacy of 
fresh frozen plasma and prothrombin complex-study design and protocol. Int J Stroke 2011;6(03):271-277

15 Sykora M, Diedler J, Poli S, et al. Autonomic shift and increased susceptibility to infections after acute intracerebral hemorrhage. Stroke 2011;42(05):1218-1223

16 Hanley JA, McNeil BJ. The meaning and use of the area under a receiver operating characteristic (ROC) curve. Radiology 1982; 143(01):29-36

17 Krouwer JS. Why Bland-Altman plots should use $\mathrm{X}$, not $(\mathrm{Y}+\mathrm{X}) / 2$ when X is a reference method. Stat Med 2008;27(05):778-780

18 Bossuyt PM, Cohen JF, Gatsonis CA, Korevaar DASTARD group. STARD 2015: updated reporting guidelines for all diagnostic accuracy studies. Ann Transl Med 2016;4(04):85

19 Eller T, Busse J, Dittrich M, et al. Dabigatran, rivaroxaban, apixaban, argatroban and fondaparinux and their effects on coagulation POC and platelet function tests. Clin Chem Lab Med 2014;52 (06):835-844
20 Seyve L, Richarme C, Polack B, Marlu R. Impact of four direct oral anticoagulants on rotational thromboelastometry (ROTEM). Int J Lab Hematol 2018;40(01):84-93

21 Takeshita S, Tanaka KA, Sawa T, Sanda M, Mizobe T, Ogawa S. Whole blood point-of-care testing for incomplete reversal with idarucizumab in supratherapeutic dabigatran. Anesth Analg 2020;130(02):535-541

22 Akman N, Braunschweig T, Honickel M, et al. Reversal of dabigatran by intraosseous or intravenous idarucizumab in a porcine polytrauma model. Br J Anaesth 2018;120(05):978-987

23 Taune V, Skeppholm M, Ågren A, et al. Rapid determination of anticoagulating effects of dabigatran in whole blood with rotational thromboelastometry and a thrombin-based trigger. J Thromb Haemost 2018;16(12):2462-2470

24 Harenberg J, Schreiner R, Hetjens S, Weiss C. Detecting anti-IIa and anti-Xa direct oral anticoagulant (DOAC) agents in urine using a DOAC dipstick. Semin Thromb Hemost 2019;45(03):275-284 